\title{
Amertume et fractions azotées de fromages à pâte molle de type camembert : rôle de l'association de Penicillium camemberti avec Geotrichum candidum
}

\author{
P Molimard 1, I Lesschaeve 1, I Bouvier 2, L Vassal 3, \\ P Schlich ${ }^{1}$, S Issanchou ${ }^{2}$, HE Spinnler ${ }^{1}$
}

\author{
${ }^{1}$ Laboratoire de recherches sur les arômes, INRA, BP 1540, F-21034 Dijon Cedex; \\ 2 Sanofi Bio-Industries, division Cultures \& Enzymes, 66, av Marceau, F-75008 Paris ; \\ 3 Station de recherches laitières, INRA, F-78352 Jouy-en-Josas Cedex, France
}

(Reçu le 16 mars 1994; accepté le 16 juin 1994)

\begin{abstract}
Résumé - Seize fromages expérimentaux de type camembert, fabriqués avec 4 souches de Penicillium camemberti utilisées seules ou associées avec 3 souches de Geotrichum candidum, ont été évalués par un jury de 18 sujets sélectionnés et entrainés. Parallèlement, ces produits ont été soumis à des dosages d'azote total, d'azote soluble à pH 4,6 et dans l'acide phosphotungstique ainsi que d'azote ammoniacal. Les activités aminopeptidasique et protéasique des 7 souches ont également été mesurées. L'amertume des produits peut être corrélée avec leur concentration en azote soluble à pH 4,6 et en peptides. De même, le caractère ammoniaqué des produits est significativement lié à la teneur en ammoniaque libre. Les produits ensemencés avec Penicillium camemberti seul sont jugés plus amers, ils possèdent également les plus fortes teneurs en azote soluble à $\mathrm{pH} 4,6$ et en peptides. Geotrichum candidum a un fort pouvoir désamérisant qui semble dû à une activité aminopeptidasique plus intense que celle de Penicillium camemberti. Les concentrations en azote total et azote soluble dans l'acide phosphotungstique des 16 produits ne sont pas significativement différentes.
\end{abstract}

camembert / fraction azotée / amertume / Penicillium camemberti / Geotrichum candidum

Summary - Bitterness and nitrogen fractions of mold ripened cheese of camembert type: impact of the association of Penicillium camemberti with Geotrichum candidum. Sixteen experimental camembert cheeses were prepared with 4 strains of Penicillium camemberti used alone or in mixed culture with 3 strains of Geotrichum candidum. They were tasted by a panel of 18 selected and trained judges. Nitrogen fractions of these cheeses were analysed (total nitrogen, soluble nitrogen at $\mathrm{pH} 4.6$, soluble nitrogen in phosphotungstic acid, ammonia). Proteolytic and aminopeptidasic activities were also measured. Bitterness of cheeses is correlated with the concentration of soluble nitrogen at $\mathrm{pH} 4.6$ or concentration of peptides. The ammonia taste is also correlated with the free ammonia concentration. Cheeses inoculated with pure Penicillium 
camemberti were judged to be more bitter. They have also the highest concentrations in soluble nitrogen at $\mathrm{pH} 4.6$ and in peptides. Geotrichum candidum has a higher aminopeptidasic activity than Penicillium camemberti; it may be the pathway by which Geotrichum candidum would decrease bitterness. Total nitrogen concentration and soluble nitrogen concentration in phosphotungstic acid of the 16 cheeses were not significantly different.

camembert cheese / nitrogen fraction / bitterness / Penicillium camemberti / Geotrichum candidum

\section{INTRODUCTION}

Les caractères organoleptiques des fromages résultent du métabolisme des divers constituants du caillé. La protéolyse a un rôle important dans la genèse de la flaveur, par la libération de nombreux peptides et acides aminés, eux-mêmes précurseurs de composés aromatiques (Adda et al, 1982). Certains peptides, en particulier les peptides de faible masse moléculaire et de forte hydrophobicité, sont connus pour développer une forte saveur amère (Lemieux et Simard, 1992). L'amertume, lorsqu'elle est trop prononcée, est considérée comme un défaut grave. Ce défaut est le plus fréquent dans le cas des fromages à pâte molle ou à pâte persillée (Pélissier et al, 1974).

Un grand nombre de facteurs interviennent sur la protéolyse et donc sur le développement de l'amertume dans les fromages (Lemieux et Simard, 1991). Dans le cas du camembert, l'activité protéolytique de Penicillium camemberti semble être le principal agent du développement de l'amertume, lorsque sa croissance n'est pas limitée (Ribadeau-Dumas, 1984 ; Vassal et Gripon, 1984). Les protéases des levains lactiques peuvent également agir sur l'amertume des produits (Martley, 1975). L'influence de la présure est, dans ce type de fromages, moins importante (Martley, 1975 ; RibadeauDumas, 1984 ; Vassal et Gripon, 1984).

Plusieurs solutions ont été proposées pour limiter le développement de l'amertume dans les camemberts:
- lincubation des fromages dans une atmosphère légèrement ammoniacale (RibadeauDumas, 1984 ; Vassal et Gripon, 1984) :

- l'ensemencement des fromages avec Geotrichum candidum (Mourgues et al, 1983 ; Vassal et Gripon, 1984).

Ces traitements ont pour effet de limiter le développement de Penicillium camemberti et ainsi d'avoir une protéolyse moins intense ce qui réduit fortement le caractère amer des fromages.

L'objectif de notre travail est d'explorer l'impact de diverses associations de Penicillium camemberti avec Geotrichum candidum sur les qualités organoleptiques de fromages de type camembert, en particulier sur l'amertume. II nous a paru intéressant d'approfondir le rôle de Geotrichum candidum dans la réduction de l'amertume, par une analyse des différentes fractions azotées trouvées dans les camemberts fabriqués avec et sans Geotrichum candidum et de regarder le lien entre cette composition et les activités protéolytiques et aminopeptidasiques des souches utilisées.

\section{MATÉRIEL ET MÉTHODES}

\section{Matériel biologique}

Au cours de ce travail, nous avons utilisé 4 souches de Penicillium camemberti (Pc1, Pc2, $\mathrm{Pc} 3, \mathrm{Pc} 4)$ et 3 souches de Geotrichum candidum (Gc1, Gc2, Gc3). Gc0 est utilisé pour indiquer l'absence de Geotrichum candidum dans les fromages. Ces souches proviennent de la collec- 
tion de Sanofi Bio-Industries, Division Cultures \& Enzymes (Paris, France).

\section{Dosage des activités protéasiques neutres et aminopeptidasiques}

\section{Culture des souches}

Les souches de Penicillium camembertiet Geotrichum candidum ont été cultivées en tubes contenant $8 \mathrm{ml}$ de milieu Czapeck Trypticase (glucose : $10 \mathrm{~g}$; trypticase : $10 \mathrm{~g} ; \mathrm{KCl}: 0,5 \mathrm{~g}$; $\mathrm{MgSO}_{4} 7 \mathrm{H}_{2} \mathrm{O}: 0,5 \mathrm{~g}: \mathrm{FeSO}_{4} 7 \mathrm{H}_{2} \mathrm{O}: 0,1 \mathrm{mg}$; solution de Meyer et Knight (Meyer et Knight, 1958) : $10 \mathrm{ml}$; tampon phosphate $\mathrm{pH} 6,0(0,05$ $\mathrm{mol} / \mathrm{l})$ : qsp $1000 \mathrm{ml}$ ). Après 6 jours à $23^{\circ} \mathrm{C}$, les cultures ont été filtrées. Les aminopeptidases et les protéases neutres ont été dosées sur 5 cultures indépendantes.

\section{Mesure de l'activité aminopeptidasique}

Les aminopeptidases ont été dosées sur les extraits exocellulaires bruts par mesure de l'hydrolyse de $0,1 \mathrm{ml}$ d'une solution méthanolique de Lleucine 4-nitroanilide (LEUPA) (Merck, Darmstadt, Allemagne) $(6,4 \mathrm{mg}$ de LEUPA dans $1 \mathrm{ml}$ de méthanol) par $0,1 \mathrm{ml}, 0,5 \mathrm{ml}$ et $2,4 \mathrm{ml}$ de filtrat de culture pendant $1 \mathrm{~h}$ à $37^{\circ} \mathrm{C}$ dans du tampon phosphate $\mathrm{pH} 6,0(0,1 \mathrm{mo} / /) \mathrm{qsp} 3 \mathrm{ml}$. La réaction a été stoppée par ajout de $0,5 \mathrm{ml}$ d'acide acétique à $30 \%$. La densité optique (DO) a alors été mesurée à $410 \mathrm{~nm}$ contre un blanc préparé avec $0,1 \mathrm{ml}$ de LEUPA en méthanol et $2,9 \mathrm{ml}$ de tampon.

\section{Mesure de l'activité protéolytique à $\mathrm{pH}$ neutre}

L'hydrolyse de $1 \mathrm{ml}$ d'azocaséine (Sigma, SaintQuentin-Fallavier, France) à $4 \%$ par $0,1 \mathrm{ml}, 0,5 \mathrm{ml}$ et $1,5 \mathrm{ml}$ de filtrat de culture a été mesurée après incubation pendant $1 \mathrm{~h}$ a $37^{\circ} \mathrm{C}$ dans du tampon phosphate $\mathrm{pH} 6,0(0,1 \mathrm{~mol} / \mathrm{l}) \mathrm{qsp} 2 \mathrm{ml}$. La réaction a été stoppée par ajout de $2 \mathrm{ml}$ d'une solution de TCA à $12,5 \%$, le milieu a été centrifugé 10 min à $3000 \mathrm{rpm}$. À $1 \mathrm{ml}$ de surnageant a été ajouté $1 \mathrm{ml}$ de $\mathrm{NaOH} 0,5 \mathrm{~N}$, la DO à $440 \mathrm{~nm}$ a alors été mesurée contre un blanc contenant $1 \mathrm{ml}$ d'azocaséine, $1 \mathrm{ml}$ de tampon phosphate $\mathrm{pH} 6,0$ $(0,1 \mathrm{~mol} / \mathrm{l})$ et $2 \mathrm{ml}$ de TCA $12,5 \%$.

\section{Fabrication des fromages}

Chaque souche de Penicillium camembertia été utilisée seule et en association avec les 3 souches de Geotrichum candidum. Seize produits différents ont donc été fabriqués. La fabrication de ces produits a été répétée 4 fois. Elle a êté réalisée au rythme de 4 produits par semaine, formant un "bloc". La répartition des 16 produits en blocs de 4 a été planifiée de façon à ce que, grâce à 3 répétitions par fabrication, chacun des degrés de liberté des combinaisons Penicillium camemberti et Geotrichum candidum soit confondu une seule fois avec l'effet bloc. Ainsi, grâce à cette planification, aucun des effets principaux n'est confondu avec l'effet bloc. Les blocs des répétitions 1 et 4 sont identiques, la première répétition de fabrication étant destinée à l'entraînement du jury de dégustateurs.

Quarante litres de lait, standardisé à 28\% de matière grasse et pasteurisé $18 \mathrm{~s}$ à $72^{\circ} \mathrm{C}$, ont été utilisés pour obtenir un lot de 20 fromages. Le lait, refroidi a $35^{\circ} \mathrm{C}$, a été inoculé immédiatement à $2 \%$ avec un mélange contenant $5 \%$ d'une culture sur lait reconstitué $(10 \% \mathrm{p} / \mathrm{v})$ autoclavé de streptocoque lactique $\mathrm{Prot}^{+}$(souche $1139 \mathrm{G}$ ) et $95 \%$ d'une culture de streptocoque lactique Prot' (souche $1140 \mathrm{P}$ ). Ces souches proviennent de la collection du CNRZ (Jouy-en-Josas, France).

Les suspensions de spores de Penicillium camemberti et Geotrichum candidum ont été ajoutées au lait avant l'emprésurage, à raison de 2500 spores $/ \mathrm{ml}$ de lait environ $(99 \%$ de Penicillium camemberti et $1 \%$ de Geotrichum candidum lorsque ces 2 espéces sont utilisées en association).

L'emprésurage a été réalisé avec $15 \mathrm{ml} / 100 \mathrm{I}$ de présure Granday ${ }^{\$}$ à $520 \mathrm{mg} /$ de chymosine (Sanofi Bio-Industries, Paris, France). Le caillé était découpé après une première phase d'acidification. Le pH était alors compris entre 5,98 et 6,19 . Les moules étaient retournés 2 fois pendant l'égouttage. Le pH du caillé était alors compris entre 5,00 et 5,68 au premier retoumement et entre 4,71 et 5,14 au deuxième retournement.

Le lendemain, les fromages étaient salés en saumure saturée pendant $40 \mathrm{~min}$ à $15^{\circ} \mathrm{C}$. Les fromages ont ensuite été maintenus $24 \mathrm{~h}$ à $15^{\circ} \mathrm{C}$ et $85 \%$ d'humidité relative, puis affinés à $12^{\circ} \mathrm{C}$ et $95 \%$ d'humidité relative. Ils ont été emballés à l'âge de 12 jours et placés ensuite à $8^{\circ} \mathrm{C}$ et $85 \%$ d'humidité relative pendant 2 semaines. 


\section{Dosage des fractions azotées}

Pour chacune des analyses suivantes, les mesures ont été répétées 3 fois; on considère alors la moyenne des 3 valeurs.

\section{Ammoniaque}

Une électrode à ammoniaque (Orion Research Inc, Boston, MA, États-Unis) a été utilisée pour ce dosage ; $25 \mathrm{~g}$ de fromage ont été broyés (broyeur Ultra-turrax ${ }^{(1)}$ ) dans $50 \mathrm{ml}$ d'eau ultrapure (Milli$Q^{\circledR}$, Millipore corporation, Bedford, MA, ÉtatsUnis). Le broyat a été dilué 10 fois, $50 \mathrm{ml}$ de la dilution ont été basifiés avec $2 \mathrm{ml}$ de soude $5 \mathrm{~mol}$ (Prolabo, Paris, France) de façon à obtenir un $\mathrm{pH}$ supérieur à 11 , les ions ammonium sont ainsi transformés en ammoniaque. Le potentiel électrique est lu après équilibrage de l'électrode dans la solution pendant $10 \mathrm{~min}$, puis reporté sur une courbe d'étalonnage réalisée préalablement avec une solution d'ammoniaque (Prolabo) dans les mêmes conditions.

$\mathrm{Au} \mathrm{pH}$ normal du fromage, seule une partie de l'ammoniaque se trouve sous forme $\mathrm{NH}_{3}$. l'autre partie étant sous forme d'ion ammonium. Connaissant le $\mathrm{pH}$ ainsi que la concentration en ammoniaque total de nos échantillons, la teneur en ammoniaque libre a été déterminée par la relation :

$$
\mathrm{NH}_{3 \text { libre }}=\mathrm{NH}_{3 \text { total }} /(1+10(9,3-\mathrm{pH})) \text {. }
$$

Nous avons vérifié ce calcul par des dosages, sans basification préalable du broyat, sur quelques échantillons pris au hasard. Les résultats obtenus sont comparables à ceux calculés.

\section{Azote total (NT)}

À $10 \mathrm{~g}$ de fromage råpé, $40 \mathrm{ml}$ de citrate trisodique $0,5 \mathrm{~mol} / \mathrm{a} a 0^{\circ} \mathrm{C}$ ont été ajoutés. Après un broyage de $30 \mathrm{~s}$ (broyeur Ultra-turrax ${ }^{(7)}$ ), le mélange a été refroidi à $22^{\circ} \mathrm{C}$ puis ajusté à $200 \mathrm{ml}$ avec de l'eau Milli- $Q^{\circledR}$. Cette solution citratée, dans laquelle les caséines se trouvent décalcifiées, a servi au dosage de l'azote total et à la préparation des solutions d'azote soluble.

\section{Azote soluble à pH 4,6 (NS)}

À $150 \mathrm{ml}$ de la solution citratée, de l'acide chlorhydrique $1 \mathrm{~N}$ a été ajouté goutte à goutte afin d'abaisser le pH à 4,4 et de précipiter les caséines. Après 20 min d'agitation à température ambiante, cette préparation a été ajustée à $200 \mathrm{ml}$ avec de l'eau Milli-Q ${ }^{\circledR}$, avant d'être centrifugée à $5000 \mathrm{rpm}$ et $10^{\circ} \mathrm{C}$ pendant $10 \mathrm{~min}$. Le dosage de l'azote soluble a été réalisé sur le surnageant.

\section{Azote soluble dans l'acide phosphotungstique (NSAPT)}

À $25 \mathrm{ml}$ de la solution d'azote soluble, on a ajouté $12,5 \mathrm{ml}$ d'une solution d'acide phosphotungstique à $10 \%$ et $12,5 \mathrm{ml}$ d'une solution d'acide sulfurique à $25 \%$. Après $24 \mathrm{~h}$ de contact à température ambiante le précipité a été éliminé par filtration. La fraction limpide représente l'azote soluble après précipitation par l'acide phosphotungstique. Elle comprend quelques petits peptides ainsi que les acides aminés, à l'exclusion des acides diaminés.

L'azote total, l'azote soluble à pH 4,6 ainsi que l'azote soluble dans l'acide phosphotungstique ont été dosés sur un automate Gerhardt Vapodest ${ }^{\circledR} 6$ (OSI, Paris, France) selon la méthode Kjeldahl.

\section{Mesure du pH}

Le $\mathrm{pH}$ a été mesuré directement au cœur des fromages grâce à une électrode de pénétration INGOLD ${ }^{\circledR}(\mathrm{OSI})$. La mesure, répétée 3 fois, a été réalisée sur 3 fromages différents.

\section{Détermination de la teneur en matière sèche}

La teneur en matière sèche des fromages a été déterminée par dessication à $101^{\circ} \mathrm{C}$.

\section{Évaluation sensorielle}

Un jury de 18 dégustateurs, sélectionnés sur leurs capacités gustatives et olfactives parmi 118 candidats, a été mis en place. L'entraînement a duré 4 mois, à raison de 2 séances de $1 \mathrm{~h} 30$ par semaine. Les produits utilisés pour l'entraînement étaient des fromages du commerce et la 
première répétition de fabrication de nos 16 produits expérimentaux.

Les 3 répétitions de fabrication suivantes ont été dégustées à raison de 4 produits par semaine évalués chacun 2 fois, à 2 jours d'intervalle. La note moyenne des 2 évaluations a été retenue. Les ordres de présentation des 4 échantillons ont été équilibrés pour l'ordre et l'effet de report (Schlich, 1993) selon un plan d'expérience conçu sur la base de carrés latins $4 \times 4$. Un fromage de chaque produit a été analysé par un groupe de 4 sujets. Lors de ces séances d'analyse sensorielle, les sujets ont noté, pour chaque échantillon, l'intensité des descripteurs "ammoniaque" et "amer" sur une échelle continue non structurée de $130 \mathrm{~mm}$. Le système PSA ${ }^{\circledR}$ (OP\&P, Utrecht, Pays-Bas) a permis la création des questionnaires, la saisie automatique des données sensorielles par les sujets et la conversion automatique des notes en valeurs variant de 0 à 100 . Les dégustations ont eu lieu dans une salle climatisée à $20 \pm 1^{\circ} \mathrm{C}$ sous une lumière rouge. Les échantillons, identifiès par un code à 3 chiffres, étaient présentés aux dégustateurs de façon monadique. Leur température était alors de $15 \pm$ $1^{\circ} \mathrm{C}$. Les dégustateurs écroûtaient le fromage avant la dégustation, selon un protocole bien défini.

\section{Analyses statistiques}

Tous les traitements statistiques ont été menés sous le logiciel SAS ${ }^{\circledR}$ (SAS Institute Inc, Cary, NC, États-Unis).

Deux analyses de la variance sur les données sensorielles ont été effectuées au moyen de la procédure GLM de SAS/STAT ${ }^{\circledR}$ (1989), suivies d'un test de comparaison des moyennes de Scheffé. Une première analyse s'est intéressée aux facteurs "sujet" et "produit" ainsi qu'à leur interaction "produit $x$ sujet". Une seconde analyse a étudié l'effet des facteurs "sujet", "Penicillium camemberti", "Geotrichum candidum" et l'interaction «Penicillium camemberti x Geotrichum candidum". L'utilisation de la procédure GLM a été motivée par la présence de données manquantes. En effet, un des 16 produits a été évalué seulement sur 2 répétitions de fabrication et un des 18 sujets a été absent lors d'une semaine de dégustation.

Deux analyses de la variance sur les données physico-chimiques ont été effectuées au moyen de la procédure ANOVA de SAS/STAT ${ }^{\circledR}$ (1989), suivie d'un test de comparaison des moyennes de Student-Newman-Keuls. La première analyse a porté sur le facteur "produit", la seconde sur les facteurs «Penicillium camemberti», "Geotrichum candidum" et sur leur interaction.

La recherche des corrélations entres les variables sensorielles amer ou ammoniaque et les variables physico-chimiques a été menée au moyen de la procédure REG de SAS/STAT ${ }^{\circledR}$ (1989).

\section{RÉSULTATS}

\section{Extrait sec et $\mathrm{pH}$ des fromages}

La teneur en matière sèche des fromages se situe entre 45,2 et $50,6 \%$ avec une moyenne de $47,5 \pm 1,6 \%$. Leur $\mathrm{pH}$ est compris entre 5,8 et 6,4 avec une moyenne de $6,1 \pm 0,2$. II n'y a aucune différence significative entre les fromages pour ces 2 paramètres.

\section{Dosage des fractions azotées}

Les résultats concernant les dosages de l'ensemble des fractions azotées, sur 4 répétitions de fabrication, sont résumés dans le tableau I.

La teneur en azote total des 16 produits expérimentaux évolue entre 30,9 et $34,3 \mathrm{~g}$ par $\mathrm{kg}$ de poids frais. L'analyse de la variance à un facteur (produit) ne révèle pas de différence significative $(P<0,05)$ entre les produits, aussi bien pour les teneurs en azote total qu'en azote soluble dans l'acide phosphotungstique. II existe, pour les teneurs en azote soluble à $\mathrm{pH} 4,6$ et en peptides, une différence significative $(P<0,05)$ entre les 16 produits. Les teneurs les plus élevées sont observées avec le produit $\mathrm{Pc}^{*} \mathrm{GcO}(14,2 \mathrm{~g} / \mathrm{kg}$ de poids frais pour l'azote soluble à $\mathrm{pH} 4,6$ et $10,1 \mathrm{~g} / \mathrm{kg}$ de poids frais pour les peptides). Les teneurs 
Tableau I. Fractions azotées des produits expérimentaux sur 4 répétitions de fabrication. Notes «amer" et «ammoniaquen sur 3 répétitions de fabrication,

\begin{tabular}{|c|c|c|c|c|c|c|c|c|}
\hline Produits & $\begin{array}{l}\mathrm{NT}^{1}(\sigma) \\
\mathrm{g} / \mathrm{kg}\end{array}$ & $\begin{array}{l}\mathrm{NS}^{2}(\sigma) \\
\% \mathrm{NT}\end{array}$ & $\begin{array}{l}\operatorname{NSAPT}^{3}(\sigma) \\
\% \mathrm{NT}\end{array}$ & $\begin{array}{l}\mathrm{P}^{4}(\sigma) \\
\% \mathrm{NT}\end{array}$ & $\begin{array}{l}\mathrm{NH}_{3} \mathrm{~T}^{5}(\sigma) \\
\% \mathrm{NT}\end{array}$ & $\begin{array}{l}\mathrm{NH}_{3} \mathrm{~L}^{6}(\sigma) \\
\mathrm{mg} / \mathrm{kg}\end{array}$ & $\operatorname{Amer}^{7}(\sigma)$ & Ammoniaque $^{8}(\sigma)$ \\
\hline $\mathrm{Pcl}^{*} \mathrm{GcO}$ & $32,6(2,4)^{a}$ & $32,3(3,2)$ ab & $11,1(1,7)^{a}$ & $21,2(1,3)^{D}$ & $08,3(2,5)^{d}$ & $05,1(1,5)^{a}$ & $48,1(5,1)^{\mathrm{Bcd}}$ & $13,1(2,8)^{\mathrm{C}}$ \\
\hline $\mathrm{Pcl}^{*} \mathrm{Gcl}$ & $32,4(2,1)^{\mathrm{a}}$ & $31,0(2,4)$ ab & $10,7(0.6)^{a}$ & $20,3(1,6)^{b}$ & $09,1(3,0)^{c d}$ & $02,4(0,8)^{a}$ & $53,8(7,0)^{\mathrm{cd}}$ & $21,5(7,6)^{\mathrm{bc}}$ \\
\hline $\mathrm{Pcl}^{*} \mathrm{Gc} 2$ & $31,6(1,2)^{a}$ & $32,4(2,9)$ ab & $11,6(2,7)^{a}$ & $20,8(1,8)^{b}$ & $11,1(1,9)$ bcd & $03,9(3,4)^{\mathrm{a}}$ & $39,5(4,5)^{\mathrm{cd}}$ & $26,8(8,8)^{a b c}$ \\
\hline Pcl"Gc3 & $33,6(2,2)^{a}$ & $33,1(6,5)$ ab & $11,5(1,5)^{a}$ & $21,6(3,9)$ ab & $17,2(3,1)^{\text {a }}$ & $02,6(3,7)^{\mathrm{a}}$ & $40,7(11,2)^{\mathrm{cd}}$ & $23,4(10,6)^{b c}$ \\
\hline $\mathrm{Pc} 2 * \mathrm{GcO}$ & $32,4(2,1)^{a}$ & $44,4(9.5)^{a}$ & $12,9(3,3)^{\mathrm{a}}$ & $31,5(5,0)^{a}$ & $14,2(0,8)^{\text {abcd }}$ & $01,0(0,5)^{a}$ & $71,4(6,1)^{\mathrm{a}}$ & $41,2(14,7)^{\mathrm{a}}$ \\
\hline $\mathrm{Pc} 2{ }^{*} \mathrm{Gcl} 1$ & $34,3(2,6)^{\mathrm{a}}$ & $34,7(8,4)^{a b}$ & $12,2(2.5)^{a}$ & $22,4(6.2)^{\text {ab }}$ & $15,6(4,0)$ ab & $05,7(0,8)^{\mathrm{a}}$ & $38,6(4,3)^{\text {cde }}$ & $31.6(20.1)^{\mathrm{ab}}$ \\
\hline $\mathrm{Pc}^{*}{ }^{\mathrm{Gc} 2} 2$ & $33,4(1,9)^{a}$ & $35,9(7,1)$ ab & $09,5(3,1)^{\mathrm{a}}$ & $26,4(3,4)$ ab & $12,1(5,8)^{\text {abcd }}$ & $11,1(4,5)^{\mathrm{a}}$ & $33,2(13,7)^{\text {def }}$ & $27,2(6,7)^{\text {abc }}$ \\
\hline $\mathrm{Pc} 2{ }^{*} \mathrm{Ge} 3$ & $33,2(1,4)^{a}$ & $32,8(7,3)^{\text {ab }}$ & $10,8(2.6)^{a}$ & $21,9(4,2)$ ab & $11.5(0,9)^{\text {abcd }}$ & $04,1(5.1)^{\mathrm{a}}$ & $22,9(6,4)^{\text {efg }}$ & $15,8(4,1)^{\mathrm{bc}}$ \\
\hline $\mathrm{Pc}^{*} \mathrm{GcO}$ & $33,4(0,8)^{a}$ & $28,1(4,8)^{b}$ & $09,5(2,0)^{a}$ & $18,6(3,6)^{b}$ & $11,2(1,0)$ abcd & $04,9(2,8)^{a}$ & $57,7(10,2)^{\mathrm{ab}}$ & $20,3(4,4)^{b c}$ \\
\hline $\mathrm{Pc} 3^{*} \mathrm{Gc} 1$ & $34,2(2,4)^{a}$ & $31,2(5,1)$ ab & $10,4(2,2)^{a}$ & $19,6(0,6)^{\text {ab }}$ & $14,6(11,3)^{a b c}$ & $08,8(4,5)^{\mathrm{a}}$ & $26,7(8,0)^{\text {def }}$ & $19,0(16,3)^{\mathrm{bc}}$ \\
\hline $\mathrm{Pc}^{*} \mathrm{Gc} 2$ & $34,3(2.5)^{\mathrm{a}}$ & $29,5(4.5)$ ab & $10,6(1.6)^{a}$ & $18,6(2,9)^{\mathrm{b}}$ & $13,5(4,1)$ abcd & $15,0(11,0)^{\mathrm{a}}$ & $22,3(3,8)^{\mathrm{fg}}$ & $23.4(12,8)^{b c}$ \\
\hline $\mathrm{Pc}^{*} \mathrm{Gc} 3$ & $32,7(1,5)^{\text {a }}$ & $26,9(4,5)^{b}$ & $09,4(1,7)^{\mathrm{a}}$ & $17,5(2,4)^{\mathrm{b}}$ & $11,1(1,6)$ abcd & $17,9(13,3)^{\mathrm{a}}$ & $15,7(3,3) \mathrm{g}$ & $10,9(7,0)^{\mathrm{c}}$ \\
\hline $\mathrm{Pc} 4^{*} \mathrm{GcO}$ & $33,8(2,7)^{\text {a }}$ & $36,5(10,1)^{a b}$ & $12,1(3,2)^{\mathrm{a}}$ & $24,4(6,3)^{a b}$ & $15,6(4,8)^{a b}$ & $03,7(2,2)^{\mathrm{a}}$ & $25,9(9,0)^{\text {defg }}$ & $24,1(18,3)^{\mathrm{abc}}$ \\
\hline $\mathrm{Pc} 4^{*} \mathrm{Gc} 1$ & $33,8(3,4)^{\mathrm{a}}$ & $29,4(5,65)^{b}$ & $10,0(1,0)^{a}$ & $19.4(4,1)^{b}$ & $15,3(1,7)^{a b}$ & $04,2(2,5)^{\mathrm{a}}$ & $17,1(8,7)^{\mathrm{fg}}$ & $12,6(7.29)^{\mathrm{c}}$ \\
\hline $\mathrm{Pc}^{*} \mathrm{Gc} 2$ & $32,7(1.0)^{\mathrm{a}}$ & $29,0(6.9)^{b}$ & $09,9(2,4)^{\text {a }}$ & $19.1(3.2)^{b}$ & $11,2(3,2)$ abcd & $14,2(2,2)^{\mathrm{a}}$ & $16,4(3,2) \mathrm{g}$ & $22,9(2.1)^{\mathrm{bc}}$ \\
\hline $\mathrm{Pc} 4{ }^{*} \mathrm{Gc} 3$ & $30,9(4,2)^{\mathrm{a}}$ & $28,1(3,1)^{b}$ & $09,9(1,1)^{\mathrm{a}}$ & $18,3(4,9)^{b}$ & $09,5(4,5){ }^{\mathrm{cd}}$ & $05,2(1,7)^{a}$ & $11,1(3,8)^{\top}$ & $10,6(1,7)^{\mathrm{c}}$ \\
\hline
\end{tabular}

$1 \mathrm{NT}$ : azote total en g/kg de poids frais. ${ }^{2} \mathrm{NS}$ : azote soluble à pH 4,6 en \% de l'azote total. ${ }^{3} \mathrm{NSAPT}$ : azote soluble dans l'acide phosphotungstique en \% de l'azote total. 4 P : peptides = NS - NSAPT en \% de l'azote total. ${ }^{5} \mathrm{NH}_{3} \mathrm{~T}$ : ammoniaque total en \% de l'azote total. ${ }^{6} \mathrm{NH}_{3} \mathrm{~L}$ : ammoniaque libre en $\mathrm{mg} / \mathrm{kg}$ de poids frais. ${ }^{7}$ Amer : note "amern sur une échelle de 0 à 100. ${ }^{8}$ Ammoniaque : note "ammoniaque" sur une échelle de 0 à 100 . $\sigma$ : écart type. a, b, c, d, e, f, g: groupes significativement différents au seuil de $5 \%$. I NT: total nitrogen in g/kg of fresh weight. ${ }^{2} \mathrm{NS}$ : soluble nitrogen at $\mathrm{pH} 4.6$ in \% of total nitrogen. ${ }^{3}$ NSAPT : soluble nitrogen in phosphotungstic acid in \% of total nitrogen. ${ }^{4} P$ : peptides = NS - NSAPT in \% of total nitrogen. ${ }^{5} \mathrm{NH}_{3} T$ : total ammonia in \% of total nitrogen. ${ }^{6} \mathrm{NH}_{3} \mathrm{~L}$ : free ammonia in mg/kg of fresh weight. ${ }^{7}$ Amer : bitter score on a 0 to 100 scale. ${ }^{8}$ Ammoniaque : Ammonia score on a 0 to 100 scale. $\sigma$ : standard deviation. $a, b, c, d, e, f, g$ : significant different groups at the error level of $5 \%$. 
les plus basses en azote soluble à pH 4,6 sont rencontrées chez les produits $\mathrm{Pc4}{ }^{\star} \mathrm{Gc1}$, $\mathrm{Pc}^{*} \mathrm{Gc} 2, \mathrm{Pc}^{\star}{ }^{*} \mathrm{Gc} 3, \mathrm{Pc}^{*} \mathrm{Gc0}, \mathrm{Pc}^{*} \mathrm{Gc} 3$ et les teneurs les plus faibles en peptides chez les produits $\mathrm{Pc1}{ }^{\star} \mathrm{Gc0}, \mathrm{Pc1}{ }^{\star} \mathrm{Gc1}, \mathrm{Pc1}^{\star} \mathrm{Gc2}$, $\mathrm{Pc}^{\star}{ }^{\star} \mathrm{Gc} 1, \mathrm{Pc}^{\star} \mathrm{Gc} 2, \mathrm{Pc}^{\star} \mathrm{Gc3}_{3}, \mathrm{Pc}^{\star} \mathrm{Gc0}$, $\mathrm{Pc}^{*} \mathrm{Gc} 2$ et $\mathrm{Pc}^{*} \mathrm{Gc}$.

L'analyse de la variance à 2 facteurs (Penicillium camemberti et Geotrichum candidum) met en évidence un effet significatif $(P<0,05)$ de la souche de Penicillium camembertisur les teneurs en azote soluble à $\mathrm{pH} 4,6$ et en peptides. Les concentrations sont plus élevées en présence de la souche Pc2, alors qu'il n'y a pas de différence entre les souches Pc1, Pc3 et Pc4. L'effet de la souche de Geotrichum candidum est moins significatif (tableau I). Seule, l'absence de Geotrichum candidum $(\mathrm{GcO})$ donne des produits plus riches en azote soluble à $\mathrm{pH}$ 4,6 au seuil de $8,4 \%$ et plus riches en peptides au seuil de $6,4 \%$ qu'en présence de la souche Gc3.

Les 16 échantillons renferment de 2,7 à $5,6 \mathrm{~g}$ d'ammoniaque par $\mathrm{kg}$ de fromage frais, soit $8,3 \%$ à $17,2 \%$ de l'azote total. Au sein d'un même lot de produits, on observe une très faible variabilité entre les 3 fromages analysés, le coefficient de variation moyen est de $3,74 \%$. La variabilité entre les 4 répétitions de fabrication est également faible, sauf pour $\mathrm{Pc}^{*} \mathrm{Gc} 1$ ( $\mathrm{CV}=77,4 \%$ ) (tableau I). L'ammoniaque libre dans les fromages représente $0,04 \%$ à $0,21 \%$ de l'ammoniaque total, soit 1,0 à $17,9 \mathrm{mg}$ par $\mathrm{kg}$ de produit frais. L'analyse de la variance à un facteur (produit) ne permet pas de différencier de façon significative les 16 produits sur la base de leur teneur en ammoniaque libre. Par contre, si l'on s'intéresse à l'ammoniaque total, le test de StudentNewman-Keuls permet de répartir les produits en 4 groupes significativement différents $(P<0,05)$ (tableau I). Pc1 ${ }^{*}$ Gc3 est plus riche $(5,6 \mathrm{~g} / \mathrm{kg}$ de poids frais) que $\mathrm{Pc}^{*} \mathrm{Gc0}, \mathrm{Pc}^{\star}{ }^{\star} \mathrm{Gc1}$ et $\mathrm{Pc1}{ }^{\star} \mathrm{Gc2}$. Les produits $\mathrm{Pc4} 4^{*} \mathrm{GcO}$ et $\mathrm{Pc4} 4^{*} \mathrm{Gc1}$ sont également plus riches que $P_{c 4}{ }^{\star} \mathrm{Gc} 3$. Les facteurs Penicillium camemberti et Geotrichum candidum n'ont pas d'effet significatif $(P<0,05)$ d'après les résultats de l'analyse de variance à 2 facteurs (Penicillium camembertiet Geotrichum candidum).

\section{Dosages des activités enzymatiques}

Les résultats obtenus sur 5 répétitions indépendantes mettent en évidence des activités enzymatiques différentes chez les 2 espèces (tableau II). Les souches de Penicillium camemberti présentent une activité endopeptidasique (protéase neutre) nettement plus forte que les souches de Geotrichum candidum. On observe le contraire pour l'activité aminopeptidasique, les souches de Geotrichum candidum sont plus actives, en particulier la souche Gc2.

\section{Evaluation sensorielle}

Le descripteur «amer" permet de discriminer les produits, ainsi 14 sujets sur 18 utilisent ce terme de façon discriminante $(P<$ $0,05)$ lors de la $4^{e}$ répétition de fabrication. L'interaction produit * sujet est significative à $0,3 \%$, ce qui traduit un désaccord entre les sujets sur la façon de discriminer les produits. Ce résultat est dû aux sujets qui ne discriminent pas les produits sur ce descripteur.

Les notes moyennes, affectées par le jury, sont comprises entre 11,1 pour $\mathrm{Pc} 4^{*} \mathrm{Gc} 3$ et 71,4 pour $\mathrm{Pc} 2^{\star} \mathrm{Gc0}$. La comparaison des moyennes des 3 répétitions de fabrication des 16 produits, au seuil de $5 \%$, montre que $\mathrm{Pc} 2{ }^{\star} \mathrm{GcO}$ est significativement le plus amer (tableau I). Les produits obtenus avec 2 des 4 souches de Penicillium camemberti en cultures pures (Pc2* Gc0 et $\mathrm{Pc}^{*} \mathrm{GcO}$ ) sont jugés plus amers que les produits obtenus avec les mêmes souches 
Tableau II. Activités aminopeptidasiques et protéasiques neutres des souches de Penicillium camemberti et Geotrichum candidum.

Aminopeptidasic and neutral proteolytic activities of Penicillium camemberti and Geotrichum candidum strains.

\begin{tabular}{lcc} 
Souches & $\begin{array}{c}\text { Aminopeptidase }(\sigma) \\
(U / m g)^{1}\end{array}$ & $\begin{array}{c}\text { Protéase }(\sigma) \\
(U / m g)^{1}\end{array}$ \\
\hline Pc1 & $0,334(0,007)$ & $1,528(0,120)$ \\
Pc2 & $0,137(0,011)$ & $1,380(0,120)$ \\
Pc3 & $0,092(0,024)$ & $0,869(0,120)$ \\
Pc4 & $0,228(0,012)$ & $1,020(0,036)$ \\
Gc1 & $0,422(0,012)$ & $0,078(0,005)$ \\
Gc2 & $0,738(0,027)$ & $0,101(0,008)$ \\
Gc3 & $0,430(0,005)$ & $0,106(0,009)$
\end{tabular}

$1 \mathrm{U} / \mathrm{mg}$ : hydrolyse de $1 \mathrm{mg}$ de substrat en $1 \mathrm{~h}$ par $\mathrm{ml}$ de filtrat de culture. Les valeurs sont rapportées au poids $\mathrm{sec}$ de mycelium. $\sigma$ : écart type.

$1 \mathrm{U} / \mathrm{mg}$ : hydrolysis of $1 \mathrm{mg}$ of substrate in $1 \mathrm{~h}$ per $\mathrm{ml}$ of culture filtrate. Values were related to the dry weight of mycelium. $\sigma$ : standard deviation.

associées avec une souche de Geotrichum candidum. Les produits $\mathrm{Pc} 1^{*} \mathrm{Gc}, \mathrm{Pc}^{*} \mathrm{Gc1}$, $P c 1{ }^{*} G c 2$ et $P c 1^{*} G c 3$ ne sont pas significativement différents. Les facteurs Penicillium camemberti et Geotrichum candidum, d'après une analyse de variance à 2 facteurs, ont un effet hautement significatif, de même que leur interaction. Les souches Pc1 et Pc2 ne sont pas différentes, elles donnent les produits jugés les plus amers (fig 1A). La souche Pc3 est significativement différente des autres souches, ainsi que la souche Pc4 qui développe le moins d'amertume. L'absence de Geotrichum candidum $(\mathrm{GcO})$ donne les produits significativement les plus amers (fig 1B). L'utilisation des souches Gc2 et Gc3 aboutit aux produits les moins amers.

L'intensité de la saveur amère des fromages croît avec leurs concentrations en azote soluble à pH 4,6 et en peptides (fig 2).
Les produits ensemencés avec $\mathrm{Pc2}{ }^{*} \mathrm{Gc0}$ ont obtenu les notes d'amertume les plus élevées (tableau I). Ces produits sont également les plus riches en azote soluble et en peptides. La régression linéaire, sur les données des fabrications 2,3 et 4 , selon le modèle amertume $=f($ azote soluble $)$ a un coefficient de corrélation de 0,49 pour 46 ddl. Elle est significative au seuil de $0,05 \%$ (fig 2A). La régression linéaire reliant l'amertume à la concentration en peptides a un coefficient de corrélation de $0,48(P<0,07$; $\mathrm{ddl}=46$ ) (fig 2B).

Le descripteur "ammoniaque" permet également de discriminer les produits, ainsi 12 sujets sur 18 l'utilisent de façon discriminante au seuil de $5 \%$ lors de la ${ }^{4}$ e répétition de fabrication. On note l'existence d'une interaction produit"sujet, due au fait que certains sujets discriminent les produits d'après ce descripteur et pas d'autres, il y a donc un désaccord entre les sujets. $\mathrm{Pc} 2^{*} \mathrm{Gc0}$ est le produit le plus ammoniaqué, avec une note moyenne de 41,2 . Les 16 produits se répartissent en 3 groupes (tableau I). Lorsque les souches Pc2 et Pc4 sont associées avec $\mathrm{Gc} 3$, les produits sont notés significativement moins ammoniaqués, $\mathrm{Pc}^{*} \mathrm{Gc} 3$ et $\mathrm{Pc} 4{ }^{*} \mathrm{Gc} 3$ sont les produits pour lesquels le descripteur "ammoniaque" a la note la plus faible.

L'analyse de variance à 2 facteurs montre que l'effet des facteurs Penicillium camemberti et Geotrichum candidum, n'est pas significatif au seuil de $5 \%$ (les valeurs de $P$ sont respectivement de 0,063 et 0,123 ). Les produits obtenus avec la souche Pc2 sont cependant notés plus ammoniaqués que ceux obtenus avec les autres souches de Penicillium camemberti (fig 1C). Les souches Pc1, Pc3 et Pc4 different peu. La présence de la souche Gc3 donne des produits évalués moins ammoniaqués, alors qu'il n'y a que très peu de différence entre les produits ne contenant pas de Geotrichum candidum ( $\mathrm{Gc} 0$ ) ou fabriqués avec les souches Gc1 et Gc2 (fig 1D). 

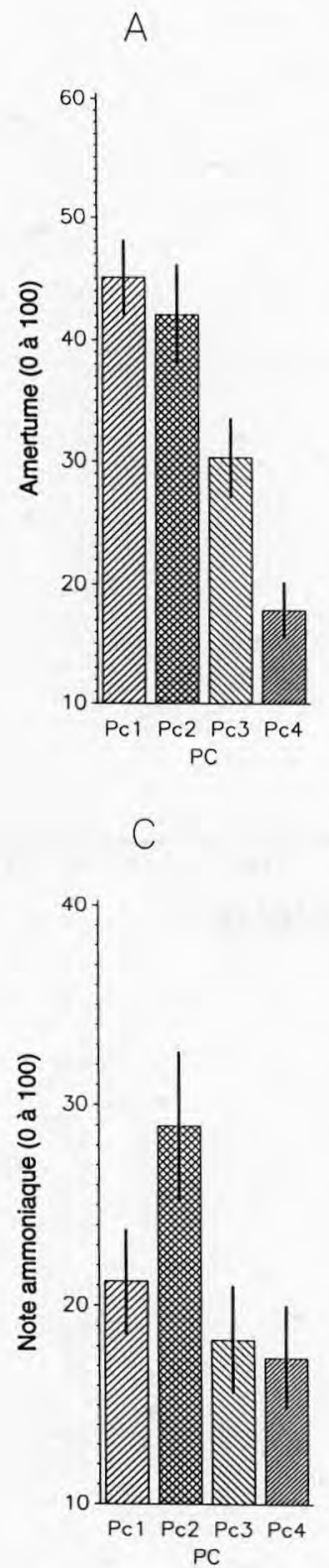
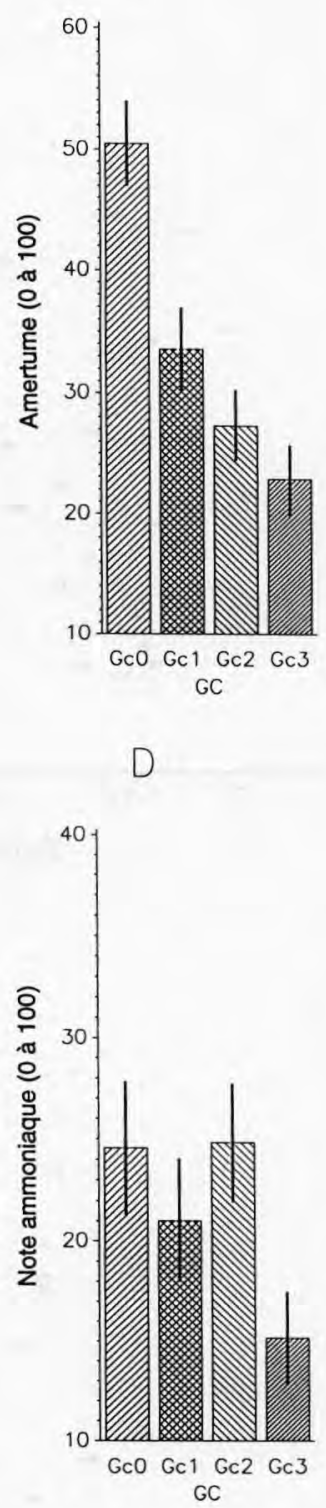

Fig 1. Amertume en fonction de la souche de Penicillium camemberti (A) et en fonction de la souche de Geotrichum candidum (B) et note ammoniaque en fonction de la souche de Penicillium camemberti (C) et en fonction de la souche de Geotrichum candidium (D). Les barres verticales représentent lintervalle de confiance à $95 \%$. A, B, C représentent les groupes significativement différents au seuil de $5 \%$. Bitterness related to the Penicillium camemberti strain $(A)$ and the Geotrichum candidum strain $(B)$ and ammonia taste related to the Penicillium camemberti strain $(C)$ and the Geotrichum candidum strain (D). The vertical lines represents the confidence interval at the level of $95 \%$. A, B, C: significantly different groups at the error level of $5 \%$. 

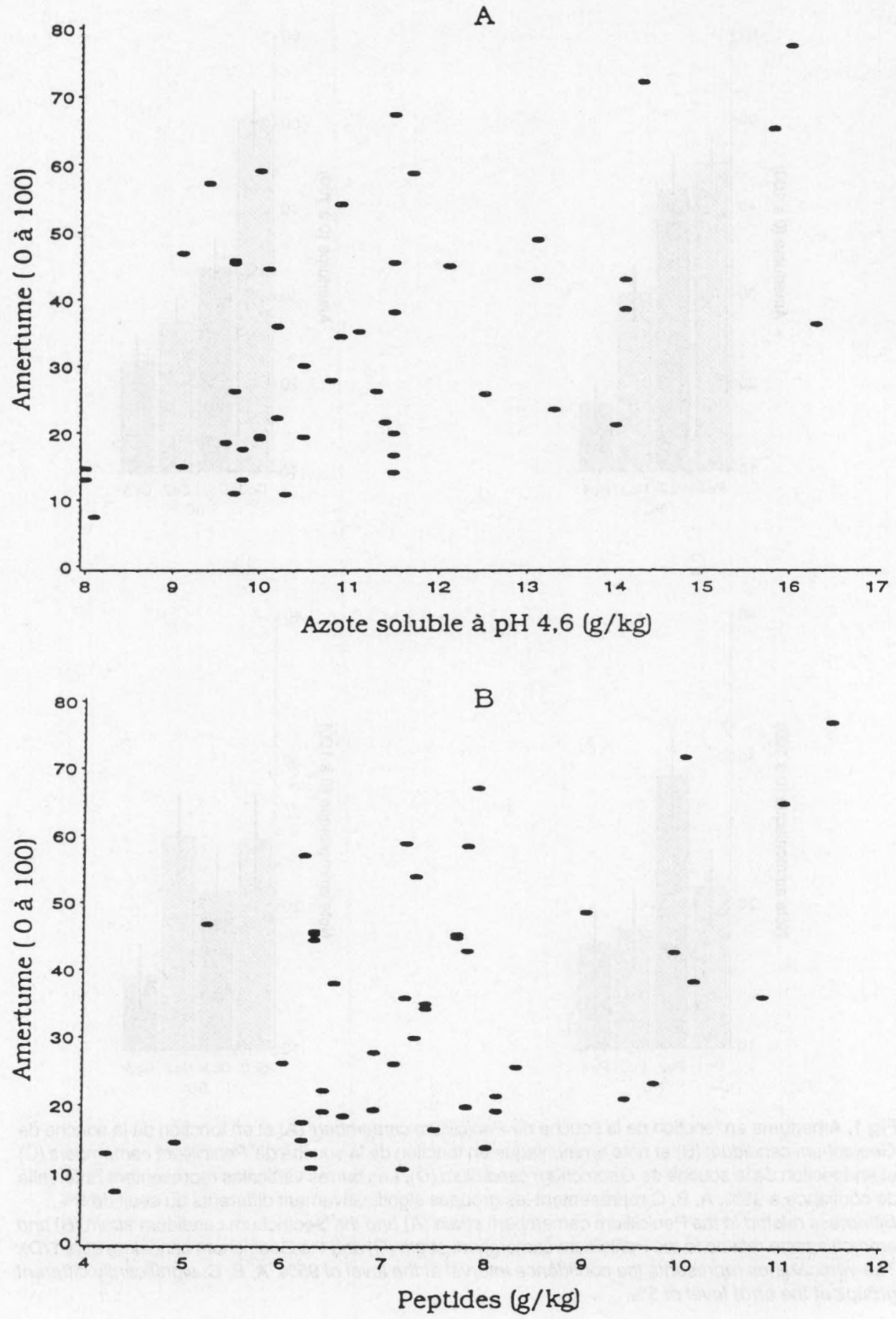
Si l'on considère les répétitions de fabrication 2,3 et 4 , la corrélation présentée figure $3 A$ entre la note "ammoniaque» et la teneur en ammoniaque libre dans les fromages $(r=0,47)$ apparaît significative $(P<0,08, \mathrm{ddl}=46)$, tout comme celle entre la note "ammoniaque" et la teneur en ammoniaque total présentée figure $3 \mathrm{~B}$ ( $r=$ 0,$52 ; P<0,02$, ddl $=46$ ).

\section{DISCUSSION}

Les corrélations, entre l'amertume et la teneur en azote soluble à pH 4,6 ainsi qu'entre l'amertume et la teneur en peptides, sont significatives malgré une importante dispersion des points observés. Cette dispersion peut s'expliquer, entre autre, par la nature de la mesure sensorielle, qui n'a pas la répétabilité ni la précision d'une mesure instrumentale, et par le fait que d'autres facteurs peuvent intervenir sur la perception de l'amertume (texture, interactions entre constituants du fromage). Une corrélation positive entre l'amertume et l'azote soluble a également été observée par Berdagué et Grappin (1988) sur des gruyères de Comté. Ces auteurs ont obtenu un coefficient de corrélation de 0,72 entre l'amertume et le Log 10 de la teneur en azote soluble dans l'eau. Dans le cas présent, l'utilisation du Log 10 de la teneur en azote soluble à pH 4,6 n'améliore pas la qualité de la corrélation. Le rôle important de certains peptides sur l'amertume des fro- mages de type camembert n'est, cependant, plus à démontrer (Lemieux et Simard, 1991, 1992). En revanche, une approximation de l'intensité de cette saveur peut être fournie par un modèle mathématique simple. Cela peut présenter un grand intérêt. II est en effet beaucoup plus aisé et moins onéreux de réaliser de simples dosages d'azote soluble que de mettre en place un jury d'analyse sensorielle. Toutefois, dans l'azote soluble il y a des peptides amers et d'autres non amers dont le ratio n'est pas apprécié par une mesure globale.

Les quantités d'ammoniaque trouvées dans nos produits sont comparables aux résultats déjà obtenus par Do Ngoc et al (1971) qui ont estimé la concentration de 10 camemberts de Normandie entre 2,7 et $3,4 \mathrm{~g} / \mathrm{kg}$ de poids frais.

De même que pour l'amertume, une approche de l'intensité du caractère ammoniaqué des fromages peut être fournie par un simple dosage de l'ammoniaque, que ce soit l'ammoniaque libre ou l'ammoniaque total.

Le seuil de perception de l'ammoniac se situe entre 1,25 et 23,9 mg/l dans l'eau selon les auteurs (Brown et al, 1968 ; Amoore et Forrester, 1976). Les quantités d'ammoniaque trouvées dans les fromages sont relativement faibles. De plus, seule une partie de cet ammoniaque est disponible. Une fraction, que l'on peut présumer non négligeable, doit être prisonnière dans la matrice. On comprend donc la difficulté, pour les dégus-

Fig 2. Amertume des 3 répétitions de fabrication des 16 produits en fonction de leurs teneurs en azote soluble à pH 4,6 (A) et en peptides (B). Amertume : note amère sur une échelle de 0 à 100. Peptides : concentration en peptides en $\mathrm{g} / \mathrm{kg}$ de poids frais. Azote soluble : concentration en azote soluble à $\mathrm{pH} 4,6 \mathrm{en} \mathrm{g} / \mathrm{kg}$ de poids frais. Les équations des droites de régression sont : amertume $=-15,4 \pm 13,3$ $+\left(4,4 \pm 1,2^{*}\right.$ azote soluble $)$ et amertume $=-5,2 \pm 11,0+\left(5,2 \pm 1,4^{*}\right.$ peptides $)$.

Bitterness of 3 cheese making replicates of the 16 cheeses related to the concentration of soluble nitrogen at pH 4.6 (A) and peptides (B). Amertume: bittemess score on a 0 to 100 scale. Peptides: peptide concentration in $\mathrm{g} / \mathrm{kg}$ of fresh weight. Azote soluble: soluble nitrogen concentration in $\mathrm{g} / \mathrm{kg}$ of fresh weight. Linear model has the following equation: amertume $=-15.4 \pm 13.3+\left(4.4 \pm 1.2{ }^{*}\right.$ azote soluble) and amertume $=-5.2 \pm 11.0+\left(5.2 \pm 1.4^{*}\right.$ peptides $)$. 

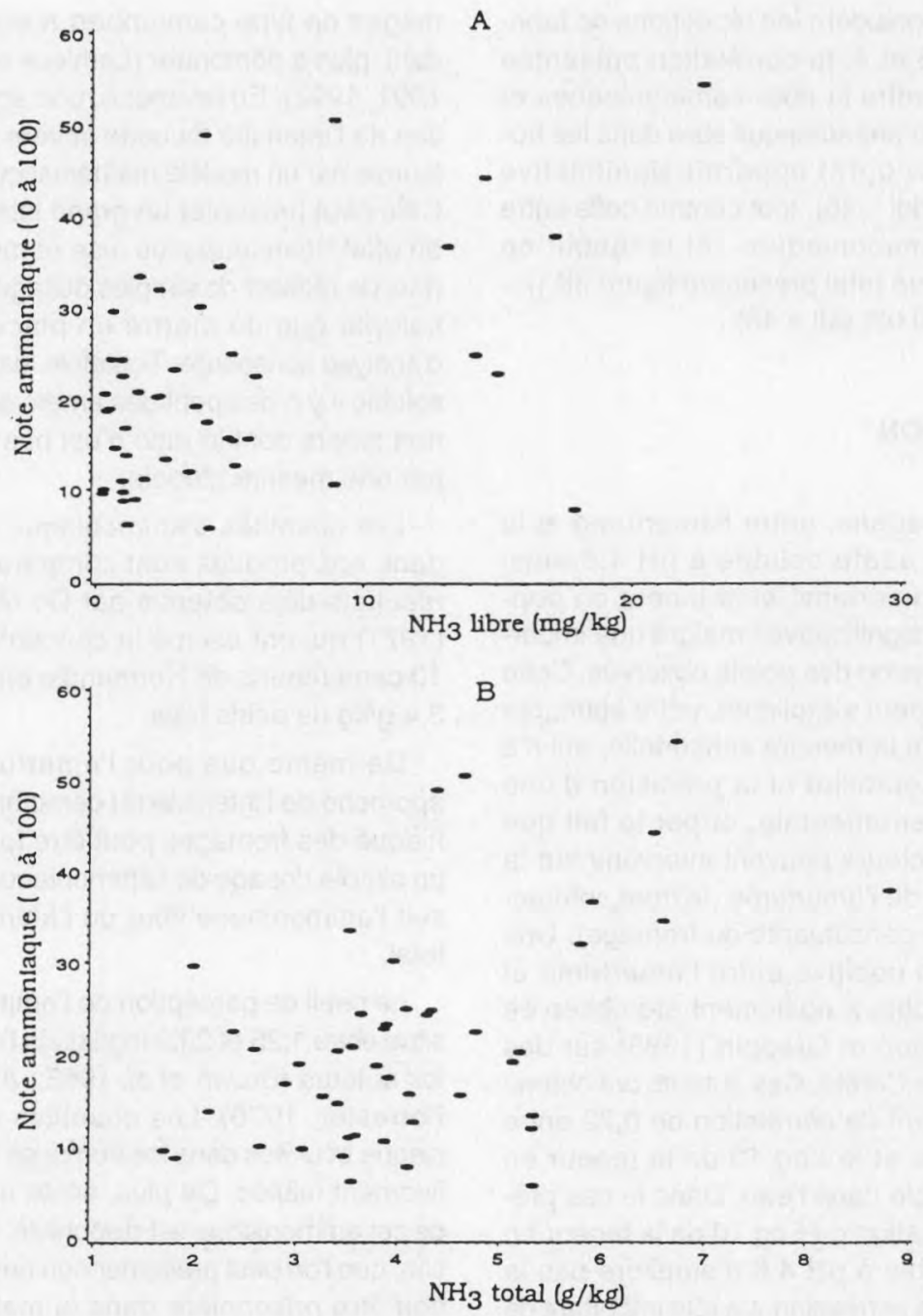

Fig 3. Note "ammoniaque» des 3 répétitions de fabrication des 16 produits en fonction de leur teneur en ammoniaque libre (A) et en ammoniaque total $(B)$. $\mathrm{NH}_{3}$ libre : concentration en ammoniaque libre en $\mathrm{mg} / \mathrm{kg}$ de poids frais. $\mathrm{NH}_{3}$ total : concentration en ammoniaque total en $\mathrm{g} / \mathrm{kg}$ de poids frais. Note ammoniaque : note ammoniaquée des produits sur une échelle de 0 à 100 . Les équations des droites de régression sont : note ammoniaque $=16,5 \pm 2,0+\left(1,0 \pm 0,3^{*} \mathrm{NH}_{3}\right.$ libre $)$ et note ammoniaque $=-7,3$ $\pm 4,7+\left(4,4 \pm 1,1 * \mathrm{NH}_{3}\right.$ total $)$.

Score of ammonia taste of 3 cheese making replicates of 16 cheeses related to the concentration of free ammonia (A) and total ammonia (B). $\mathrm{NH}_{3}$ libre: free ammonia concentration in $\mathrm{mg} / \mathrm{kg}$ of fresh weight; $\mathrm{NH}_{3}$ total: total ammonia concentration in $\mathrm{g} / \mathrm{kg}$ of fresh weight; note ammoniaque: ammonia score on a 0 to 100 scale. Linear model has the following equation: note ammoniaque $=16.5 \pm 2.0+(1.0 \pm$ $0.3{ }^{\star} \mathrm{NH}_{3}$ libre $)$ and note ammoniaque $=-7.3 \pm 4.7+\left(4.4 \pm 1.1 * \mathrm{NH}_{3}\right.$ total $)$. 
tateurs, à apprécier les faibles différences existant entre produits, ce qui peut expliquer, là aussi, la valeur relativement faible des coefficients de corrélation obtenus entre le caractère ammoniaqué des fromages et leur teneur en ammoniaque libre ou total.

Guéguen et Lenoir $(1975 a, b)$ ont observé que l'aptitude à la protéolyse de Geotrichum candidum pouvait être comparée à celle de Penicillium caseicolum; de plus le $\mathrm{pH}$ optimal de ces protéases correspond à celui des pâtes fromagères. Ces auteurs ont également observé que les souches isolées à partir de fromages à pâte molle ont, pour la plupart, une aptitude à la protéolyse plus marquée que celles isolées à partir de pâtes pressées.

II apparait clairement que Geotrichum candidum est capable d'améliorer les qualités organoleptiques des fromages par son action sur le métabolisme des protéines. II semble, à la vue de nos résultats, qu'il y ait dans les produits ensemencés avec Penicillium camembertiseul, une activité endoprotéasique plus active que l'activité exopeptidasique, ce qui conduit à une accumulation de peptides. La présence de Geotrichum candidum a pour effet de réduire la quantité d'azote soluble et de peptides. La très nette diminution de l'amertume observée confirme les résultats de Mourgues et al (1983). L'activité protéasique globale de Geotrichum candidum, génératrice de peptides, est beaucoup plus faible que celle de Penicillium camemberti. Au contraire, son activité aminopeptidasique globale est plus élevée. La désamérisation observée pourrait donc correspondre à une hydrolyse, par les aminopeptidases de Geotrichum candidum, des peptides amers générés par Penicillium camemberti.

Nous n'avons dosé que l'activité aminopeptidasique, déjà étudiée chez Geotrichum candidum (Guéguen et Schmidt, 1992), mais ce dernier possède également une activité carboxypeptidasique, encore mal connue mais qui fait actuellement l'objet d'études (Guéguen et Schmidt, 1992). Geotrichum candidum n'a par contre pas d'effet évident sur la libération de l'ammoniaque dans les fromages.

Contrairement aux résultats énoncés par Mourgues et al (1983) nous n'avons pas, par notre technique de dénombrement, mis en évidence de limitation du développement de Penicillium camembertipar Geotrichum candidum.

\section{CONCLUSION}

Un simple dosage de la concentration en azote soluble ou en peptides des fromages de type camembert peut donner une approximation du caractère amer de ceux-ci. De même, une approximation du caractère ammoniaqué peut être fournie par la quantification de l'ammoniaque présent dans les fromages. Toutefois, pour utiliser de façon plus fiable ces modèles mathématiques, une étude devrait être menée sur des échantillons présentant une gamme de variation d'intensité d'amertume et de note ammoniaque plus large que celle de nos produits, ce qui pourrait peut être améliorer la qualité des régressions entre les paramètres physico-chimiques et les paramètres sensoriels. En effet, la plupart de nos produits expérimentaux étaient homogènes pour ce caractère. Ainsi, pour des produits présentant des valeurs physico-chimiques (azote soluble à $\mathrm{pH} 4,6$, peptides, ammoniaque) très proches, l'évaluation sensorielle donne des résultats pouvant être assez différents, ce qui diminue la qualité des corrélations sensoriel-instrumental. Par ailleurs, d'autres facteurs que l'azote soluble à $\mathrm{pH} 4,6$, les peptides ou l'ammoniaque viennent également perturber la perception sensorielle de l'amertume et de la note ammoniaquée.

Les associations microbiennes, utilisées depuis longtemps pour obtenir de meilleures qualités organoleptiques, sont définies souvent de façon empirique. II ressort de ce 
travail que la connaissance des caractéristiques enzymatiques des souches de Geotrichum candidum peut permettre d'optimiser le choix d'une souche pour améliorer une caractéristique organoleptique. En effet, nous montrons que, vraisemblablement, les activités peptidasiques de Geotrichum candidum jouent un rôle majeur dans cette désamérisation des fromages. D'une façon plus générale, la caractérisation des activités enzymatiques des souches utilisées industriellement et des liens existant entre ces activités et les qualités organoleptiques pourrait sans doute permettre de gagner un temps précieux dans la mise au point et l'optimisation de la qualité de nouveaux produits.

\section{REMERCIEMENTS}

Les auteurs remercient tout particulièrement : l'équipe du laboratoire de développement de Sanofi Bio-industries, division Culture \& Enzymes, pour la production des souches; Mmes M Lacroix et C Piffaut ainsi que MM E Chaulet, $P$ Lavier et G Pitel pour leur participation technique et M Bigret pour son assistance scientifique ; la société Sanofi Bio-industries, division Cultures \& Enzymes, le ministère de l'Enseignement supérieur et de la Recherche ainsi que la région Bourgogne pour leur soutien financier.

\section{RÉFÉRENCES}

Adda J, Gripon JC, Vassal L (1982) The chemistry of flavour and texture generation in cheese. Food Chem 9, 115-129

Amoore JE, Forrester LJ (1976) Specific anosmia to trimethyamine: the fishy primary odor. $J$ Chem Ecol 2, 49-56

Berdagué JL, Grappin R (1988) Affinage et qualité du Gruyère de Comté. VI. Caractéristiques sensorielles des fromages. Lait $68,189-204$
Brown KS, Maclean CM, Robinette RR (1968) The distribution of the sensitivity to chemical odors in man. Hum Biol 40, 456-472

Do Ngoc M, Lenoir J, Choisy C (1971) Les acides aminés libres de fromages affinés de camembert, SaintPaulin et Gruyère de Comté. Rev Lait Fr 288, 447 462

Guéguen M, Lenoir J (1975a) Aptitude de l'espèce Geotrichum candidum à la production d'enzymes protéolytiques. Lait 55, 145-162

Guéguen M, Lenoir J (1975b) Aptitude de l'espèce Geotrichum candidum à la production d'enzymes protéolytiques. Note complémentaire. Lait 55, 621 629

Guéguen M, Schmidt JL (1992) Les levures et Geotrichum candidum. In: Les groupes microbiens d'intérêt laitier (Cepil, ed) Cepil, Paris, France

Lemieux L, Simard RE (1991) Bitter flavour in dairy products. I. A review of the factors likely to influence its development, mainly in cheese manufacture. Lait $71,599-636$

Lemieux L, Simard AE (1992) Bitter flavour in dairy products. II. A review of peptides from caseins: their formation, isolation and identification, structure masking and inhibition. Lait 72, 355-382

Martley FG (1975) Comportement et rôle des streptocoques lactiques du levain en fabrication de camembert. Lait 55, 310-323

Meyer E, Knight SG (1958) Studies on the nutrition of Penicillium roqueforti. Appl Microbiol 6, 174-178

Mourgues R, Bergère JL, Vassal L (1983) Possibilité d'améliorer les qualités organoleptiques des fromages de camembert gráce à l'utilisation de Geotrichum candidum. Tech Lait 978, 11-15

Pélissier JP, Mercier JC, Ribadeau-Dumas B (1974) Problème de l'amertume dans les fromages - résultats d'une enquête. Rev Lait Fr 325, 820-821

Ribadeau-Dumas B (1984) Maîtrise de l'affinage des fromages de type camembert. Lait 64, 448-468

SAS Institute Inc (1989) SAS/STAT ${ }^{\circ}$ user's guide. Version 6, Fourth Edn. Vol 1 and 2, SAS Institute Inc, Cary, NC, USA

Schlich P (1993) Uses of change over designs and repeated measurements in sensory and consumer studies. Food Qual Pref (in press)

Vassal L, Gripon JC (1984) L'amertume des fromages à pâte molle de type camembert : rôle de la présure et de Penicillium caseicolum, moyens de la contrôler. Lait 64, 397-417 